Die Zusammensetzung zeigte sich:

$$
\begin{array}{lrrr} 
& & \text { berechnet: } & \text { gefunden : } \\
\mathrm{As}^{2} \mathbf{0}^{5}=1436,48 & 28,59 & 28,55 \\
2 \mathrm{NaO} & =774,34 & 15,42 & 15,60 \\
25 \mathrm{aq} & =2812,50 & 55,99 & 55,86 \\
\cline { 2 - 4 } & 5023,32 & 100,00 & 100,01 .
\end{array}
$$

Bei $0^{\circ}$ und auch noch bei einigen Graden darunter krystallisirtes Salz ergab 56,58, 56,23 und 56,22 Proc. Wassergehalt. Die Formel $\mathrm{As}^{2} \mathrm{O}^{5}, 2 \mathrm{NaO}+26 \mathrm{aq}$ würde aber 56,96 Proc., diejenige mit 27 aq aber 57,88 Proc. Wasser erfordern; demnach enthält das bei niederen Temperaturen krystallisirte Salz auch nicht mehr als 25 Atome Wasser. (Erdm. Journ. für prakt. Chem. 1852. Bd.56. p. 30.) R.

\title{
Ueber das Wasser des todten Meeres und des Jordans.
}

Boutron-Chalard and Henry haben das Wasser des todten Meeres analysirt. Dieses wurde ihnen wohl verwahrt von dem Consul des Jerusalemer Districts zugesandt. Derselbe schöptte es den 2. April 1850. Benannte Chemiker widmeten sich dieser Untersuchung mit grossem Eifer und zwar, um der auffallenden Verschiedenheit, die sie in den Resultaten Anderer entdeckten, auf die Spur zu kommen. Der Bericht über eine Excursion an den Ufern des todten Meeres - dem Institut am 22. August 1851 vorgelesen - war den Herren Verfassern ein treffliches Hülismittel zur Erweiterung ihrer Arbeiten.

Die Ausdunstungen des todten Meeres sind nicht tödtlich, da Vögel dariber wegfliegen und Wasservögel lustig darauf herumschwimmen. Der Untergang der fünf Städte in der Gegend des todten Meeres, wovon in der Bibel die Rede ist, muss vulkanischen Eruptionen, die in diesem Theile Syriens mehrmals vorfielen, zugeschrieben werden. Nach einer von Lavoisier, Macquer und $S$ a ge angestellten Analyse enthielten 100 Theile des in Rede stehenden Wassers $44 \frac{3}{8}$ Theile Salzes, nämlich:

$$
\begin{array}{r}
\text { Natron-Seesalz...6 } \\
\text { Erdiges Seesalz } . .38 \frac{1}{4} \text { Theile } \\
\text { Sa. } 4 \frac{1}{8} \text { Theile. }
\end{array}
$$

M al te Brun sagt in seinen Annales des voyages: Das Eigengewicht des Wassers aus dem todien Meere beträgt 1,214. Es ist vollkommen klar. Reagentien verrathen darin Salzsäure und Schwefelsaure. Alaunerde fehlt. Es verändert das Lackmuspapier nicht. 100 Theile desselben enthalten: 


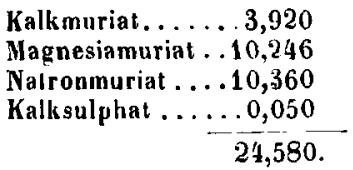

Obige Salze also machen den vierten Theil des Wassers aus. Gordo n erzahlt, dass man, ohne schwimmen zu können, davon getragen wird. Klaproth, welcher das Wasser des todten Meeres 1809 untersuchte, giebt das Eigengewicht desselben zu 1,248 an, er fand in 100:

$$
\begin{array}{r}
\text { Kalk muriat. . . . .10,60 } \\
\text { Magnesiamuriat . .24,20 } \\
\text { Natronmuriat . . . .7,80 } \\
42,60 .
\end{array}
$$

Gay Lussac, welcher das ibm vom Grafen Forbin überreichte und von demselben selbst geschöpfte Wasser des todten Meeres untersuchte, fand sein Eigengewicht 1,228 und bekam von 100 26,24 Theile eines Ruickstandes, welcher aus Natrium-, Calcium-, Magnesium- und Kaliumchlorid mit wenigem Kalksulphat bestanden.

In dem 55. Theile der Annales de Chimie et de Physique 1821 befindet sich die Analyse des Wassers aus dem todten Meere von G. G. Gmelin. Das Eigengewicht betrug nach demselben 1,212; das Resultat war in 100 folgendes:

$$
\begin{aligned}
& \text { Chlorid des Calciums.......3,2141 } \\
& \text { " " Magniums.....11,7734 } \\
& \text { " "Natriums......7,0777 } \\
& \text { " " Kaliums. ......, "738 } \\
& \text { " "Alumiums...... } 0,0896 \\
& \text { " "Mlangans.......0,2117 } \\
& \text { " "Ammoniums...0,0075 } \\
& \text { Bromid des Nagniums . . . . . . 0,4393 } \\
& \text { Kaliumoxydsulphat . . . . . 0,0527 } \\
& \text { 24,5398. }
\end{aligned}
$$

In den Annales des mines von 1840 ist noch zweier Analysen erwähnt, die eine von Ivanof, die andere von Apjohn; aber diese Nänner operirten auf zu geringe Mengen des Wassers, als dass man ihrem Resultat trauen könnte. Da die Analysen eines Lavo isier, Klaproth's Gmelin's und Gay Lussac's unter einander abweichen, so muss die Ursache davon nur besonderen Umständen und keiner Ungenauigkeit zugeschrieben werden. Das Wasser, welches B outron-Chal ard und Henry erhielten, war in einer Blechflasche mit verlötheter Mündung enthalten. Filtrirt zeigte es folgende Eigenschaften: 
Es war geruchlos, schmeckte salzig-bitter und trübte sich in der Hitze ocherfarbig. Das Eigengewicht desselben war 1,099. Gegen Lackmuspapier verhiett es sich indifferent. doch bewirkte es in der Veilchentinctur einen Blick in das Grünliche. Mit Säuren brausete es nicht. Bariumsalze trubten es etwas, Silbersalze sehr stark mit einem röthlichen Präcipitate. Phosphate verriethen die Gegenwart von Calcium- und Magniumsalzen. Die mit etwas Schwefelwasserstoff versetzte Auflösung des Eisenoxydsulphats verursachte eine graue Trühung darin, Alkohol, wie auch Seifenauflösung eine weisse. Die fast zur Trockne abgerauchte Mutterlauge des Wassers wurde mit Weingeist ausgezogen. Die Auflösung gab mit Brucinsulphat eine orangenfarbene Verbindung und Platinchlorid verrieth in dem tief abgerauchten Wasser Kali. Weil die quantitative Analyse stets unerhebliche Versuche zum Gegenstand hat, so sei hier nur der Hauptsache dabei gedacht.

100 Theile des mit etwas Salpetersäure versetzten Wassers gaben mit Silbersolution 32,7 Th. Silberchlorid. Das Filtrat mit Ammoniak im Ueberschuss erlitt zwar eine Truibung, aber eine so schwache, dass man sie nicht einmal einem Phosphate zuschreiben konnte. Mit Kali destillirt, gab es ein Destillat ohne eine Spur von Ammoniak. In der Retorte hatte sich Kalkerde und Talkerde abgesetzt. Wurde die vom Bodensalz abgesonderte Fluissigkeit bis zur Truckne abgeraucht und der Riickstand mit Weingeist behandelt, so liess sich in diesem mit Amylum kein Jod auffinden.

Ein mit Silbersalz in $60 \mathrm{Grm}$. des Wassers gewonnener Niederschlag wurde mit metallischem Zink (in stetem Ueberschuss gehalien) und reiner Schwefelsäure behandelt, dann der Niederschlag auf ein Filter geworfen, das Filtrat bis zu einem gewissen Volum abgeraucht und in einem engen Probierglase mit etwas Chlorwasser und Schwefeläther durchgeschïttelt, nun erschien jener gelb gefärbt, am Amylum war aber keine Veränderung zu bemerken; es zeigle sich weder eine blaue noch violette, noch rosenrothe Färbung an demselben, oder die drei Farben, wodurch man das Verhälıniss der Menge des Jods angedeutet sieht. Obige Erfahrung bestätigte sich durch Kaliumjodid, welches man in allmălig geringerer Quantität anwandte. Obiges Verfahren ist so einfach und so leicht auszufiuhren, dass man sich desselben gewiss in der Folge bei Mineralwasser-Analysen bedienen wird, nämlich, wenn es darauf ankommt, sich zu überzeugen, ob Bromide neben Jodiden 
vorhanden sind. Durch Uebung wird man dahin kommen, selbst die Menge dieser beiden Stoffe nach dieser Methode annähernd zu bestimmen. In 1000 Theilen des Wassers waren vorhanden:

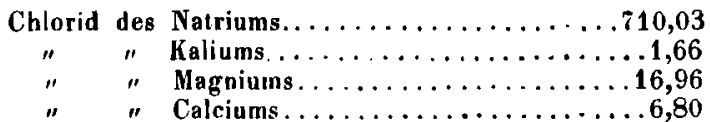

Sulphat des Natrons, Kalks und der Hlagnesia....2,33

Erdige Carbonate ..................9,53

Siliciumsâure, organische Substanzen ........8,00

Bromide, Nitrate, Fisenoxyd.............. Spuren

149,34 .

Ob das Magnesia- und Kalkcarbonal, wie auch das Sulphat während der Behandlung entstanden ist, oder ob es präexistirte, bleibı wegen der geringen Menge des behandelten Wassers unentschieden, so viel ist gewiss, dass wenn letzteres der Fall sein sollte, erstere beide als Bicarbonate vorhanden waren. Mlan würde sich iber grosse Gewichtsverschiedenbeit der Salzrückstände dieses Wassers wundern müssen, wenn sie nicht durch bekannte Umstande veranlasst wären, nämlich durch die 30 Tage lang anhaltenden Regenguisse und die dadurch entstehenden Stromergiessungen, wie auch durch die bald darauf folgende, starke Verdunstung bewirkende Temperaturerhöhung. Lavoisier, Macquer und Sage und später Kl aproth gaben einen Ruckstand von 40 Proc, Gay L u s a c und Andere von 10ّ-20 Proc. an. Obige meteorologische Wirkung, die sich in jedem Jahre wiederholt und worüber die Physiker einig sind, zeigt an, dass es wesentlich nothwendig ist, die Jahreszeit zu bemerken, in welcher das Schöpfen des Wassers geschah.

Gäbe das Wasser des todten Nleeres, wie das des Elton-Sees (im asiatischen Russland) Magnesiasulphat, so könnte man glauben, es ginge ein Phänomen darin vor, wie es Palla $\mathrm{s}$ in diesem beobachtete, nämlich, dass sich das Salz des Nachts absetzl und am Tage wieder auflöst. Nach Gay Lussac gelten die erst bemerkten Umstände beim Wasser des todien Meeres allein.

Wasser des Jordans. - Das Wasser des Jordans ist gelblich und lehmig. Dieser kleine Fluss hat nach der Jahreszeit verschiedene Tiefe und eine Breite von $\mathbf{4 0}$ bis 50 Meter. Die Ufer desselben sind mit Schilf und Binsen bewachsen. Als der Graf F orb in 1817 dem G a y Lussac Wasser aus dem todten Meere zur Analyse überreichte, hatte er demselben zu diesem $Z$ weck auch Was- 
ser des Jordans mitgebracht, aber jener begniugte sich mit Reagentienproben und fand dadurch, dass es Natrium- und Magniumchlorid und etwas weniges von Kalksulphat, wie auch Spuren von Calciumchlorid enthielt. Das Boutron-Chalard und Henry übergebene Wasser war durchsichtig, geschmacklos und der Geruch desselben erinnerte an Petroleum. Es hat ein geringes Eigengewicht, nämlich nur 1,00084. Versuche durch Reagentien ergaben darin Chloride, alkalische Carbonate, Spuren von Sulphaten und in dem Salzriickstande noch alkalisches Salz, wis es Veilchentinctur durch einen Blick ins Grinliche andeutete.

Benannte Chemiker fanden das Wasser des Jordans in 1000 Theilen zusammengesetzt aus:

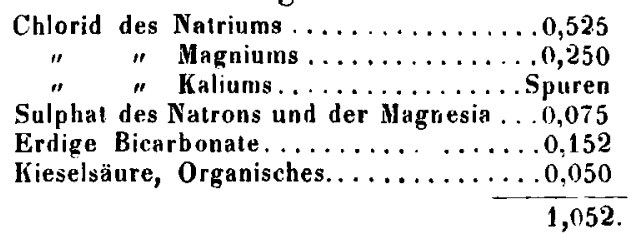

Am Ende ihrer Abhandlung theilien die Verfasser noch Versuche über zwei Fossilien mit, über ein kalkartiges und ein erdharzartiges, die sich am Ufer des todten Meeres befanden, da sie aber eben kein lnteresse darbieten, so sind sie hier weiter nicht berührt. (Journ. de Pharm. el de Chim. Mars 1852. p. 16j1.) du Ménil.

\section{Kirystallinische Beschaffenheit des Glases.}

Leydolt zu Wien hatte Achate mit Flusssaure angeätzt, davon galvanoplastisch zum Abdrucke sehr geeignete Platten hergestellt, welche die innere Structur dieser Körper sehr schön darthun, indem die krystallinischen Parthien schwerer angegriffen werden, als die amorphen und so die Verschiedenheil der angeätzten Oherflachen durch ihre eigenen treu bedingen. Auf dieselbe Weise fand Leydolt, dass alles Glas krystallinisch ist; man braucht nur Glasstreifen theilweise in das Gemisch von Flussspath und Schwefelsäure zu stellen, bis sie angeätzt sind und dann etwa so damit zu verfahren. Man kann von der Linie aus, wo die Oberfläche der Flüssigkeit den Glasstreifen berïhrt und auf der der Fluissigkeit zugekehrten Seite die krystallinische Beschaffenheit des Glases verfolgen. (Compl.rend. T.34. - Chem.-pharm. Cenirbl. 18j2. No. 25.) 